ISSN 0103-5150

Fisioter. Mov., Curitiba, v. 25, n. 2, p. 317-324, abr./jun. 2012

Licenciado sob uma Licença Creative Commons

\title{
Avaliação dos efeitos de um programa sensório-motor no padrão da marcha de idosas
}

\author{
Evaluation of the effects of a sensorial-motor \\ program in the gait pattern of aged females
}

\author{
Adriana Arruda Barbosa Rezende ${ }^{[a]}$, Iris Lima e Silva ${ }^{[b]}$, Heron Beresford ${ }^{[c]}$, Luiz Alberto Batista ${ }^{[d]}$ \\ [a] Fisioterapeuta, Mestre em Ciência da Motricidade Humana Universidade Castelo Branco, professora adjunta do Centro \\ Universitário UNIRG, Gurupi, TO - Brasil, e-mail: drikas.arruda@gmail.com \\ [b] Mestre em Ciência da Motricidade Humana Universidade Castelo Branco, pesquisadora do Laboratório de Temas \\ Filosóficos em Conhecimento Aplicado (UCB/RJ), Rio de Janeiro, RJ - Brasil, e-mail: irislimaucb@yahoo.com \\ [c] Professor titular do Programa de Pós-Graduação Stricto Sensu em Ciência da Motricidade Humana da Universidade \\ Castelo Branco (UCB/RJ), professor adjunto da Universidade do Estado do Rio de Janeiro (UERJ), Rio de Janeiro, RJ - \\ Brasil, e-mail: heronberesford@gmail.com \\ [d] Professor adjunto do Programa de Pós-Graduação Stricto Sensu em Ciência da Motricidade Humana da Universidade \\ Castelo Branco (UCB/RJ), professor adjunto da Universidade do Estado do Rio de Janeiro (UERJ), Rio de Janeiro, RJ - \\ Brasil, e-mail: bmc_ef@yahoo.com.br
}

\section{Resumo}

Introdução: Muitas alterações que ocorrem com o envelhecimento têm impacto de natureza funcional e acarretam, como uma das consequências mais importantes, distúrbios na marcha de pessoas idosas. objetivos: Avaliar a eficácia de um programa de treinamento sensório-motor, no padrão da marcha de mulheres idosas. Materiais e métodos: Atendendo-se aos critérios de exclusão e inclusão, formou-se um grupo de voluntários constituído por 15 idosas, que foram submetidas à análise do padrão da marcha por meio da videometria, cujas imagens foram processadas pelo software DgeeMe, versão 1.0. Em seguida, elas foram submetidas a um treinamento sensório-motor, por meio de um circuito que incluiu oito estações de exercícios com estímulos proprioceptivos e de equilíbrio. A frequência do treinamento foi de três sessões semanais com duração de 30 minutos, durante dois meses. Depois disso, foi realizada nova análise da marcha, considerando-se os componentes temporais e espaciais. Resultados: Apesar de melhora nos componentes temporais e espaciais da marcha, só foi verificada significância estatística para o resultado observado na 
variável comprimento da passada $(\mathrm{p}=0,02)$. Conclusão: Concluiu-se que o treinamento sensório-motor, muito embora possa melhorar o equilíbrio do indivíduo idoso, trazendo-lhe segurança para desenvolver a marcha, pode não afetar diretamente essa função nos seus aspectos biomecânicos.

Palavras-chave: Idoso. Marcha. Equilíbrio.

\begin{abstract}
Introduction: Many changes that take place with aging have an impact of a functional nature and result, as one of the most important consequences, in disorders in the gait pattern of the elderly. Objectives: To evaluate the effectiveness of a sensorial-motor program on the gait pattern of elderly females. Materials and methods: Fulfilling the exclusion and inclusion criteria, a group of volunteers was formed comprised of 15 elderly females who were submitted to analysis of the marching pattern by videometry, whose images were processed by the software DgeeMe 1.0. After that, they were submitted to a sensorial-motor training, by means of a circuit which included eight exercise stations with proprioceptive and balance stimuli. The frequency of training was three weekly sessions with duration of 30 minutes, for two months. Then, a new analysis of the gait was carried out, considering the temporal and spatial components. Results: In spite of the improvement in the temporal and spatial components of the gait, a statistical significance was verified only for the result observed in the variable of length of step $(p=0.02)$. Conclusion: It was concluded that the sensorial-motor training, even though it is able to improve the balance of the elderly subject, bringing her safety to develop the gait, can not affect this function directly in its biomechanical aspects.
\end{abstract}

Keywords: Aged. Gait. Balance.

\section{Introdução}

0 natural processo de envelhecimento é caracterizado por profundas alterações que são observadas em todos os aparelhos e sistemas orgânicos (1). Muitas dessas alterações têm impacto de natureza funcional e acarretam, como uma das consequências mais importantes, distúrbios na marcha de pessoas idosas $(2,3)$.

A marcha é um fenômeno motor complexo, resultante da interação dos sistemas neurológico, musculoesquelético, vestibular e somatossensorial. Durante o processo de envelhecimento, esses sofrem comprometimento em virtude de fatores como redução no número de células, na quantidade de água e nas proteoglicanas, aumento das células colágenas no sistema articular, diminuição da massa óssea, da massa muscular da excitabilidade muscular e da velocidade de condução nervosa e, ainda, em função de distúrbios do equilíbrio (4-6).

Assim, o idoso tem a força muscular reduzida, sofrendo, ainda, influências negativas na sua capacidade de discriminação sensorial, com consequente perda da coordenação motora, do equilíbrio e da propriocepção (5, 7-9).
Mesmo em idosos autônomos, existe a possibilidade de que essas deficiências venham a ocorrer ao longo do tempo, comprometendo o padrão da marcha e diminuindo a capacidade de deambulação do indivíduo. Uma vez que a marcha é um dos aspectos mais importantes da função física e pré-requisito para a execução das atividades de vida diária (AVDs), podem daí advir incapacidades e dependência funcional $(2,3,10-13)$, sendo importante testar-se a eficácia de intervenções que visem recuperar e prolongar o bom status operacional da marcha de pessoas idosas.

Diante disso, neste estudo teve-se como objetivo avaliar a eficácia de um programa de treinamento sensório-motor, no padrão da marcha de mulheres idosas autônomas.

\section{Materiais e métodos}

\section{Caracterização do grupo}

O grupo estudado foi constituído, voluntariamente, por 15 mulheres, sedentárias, autônomas e independentes funcionalmente, com idades entre $60 \mathrm{e}$ 
75 anos (média $=65$ ), cadastradas no Programa de Atividade Física e Reabilitação para Autonomia dos Idosos (PROAFIR) do Centro Universitário UNIRG, da cidade de Gurupi, Tocantins.

Os critérios de exclusão adotados para compor o grupo foram: marcha dependente de dispositivo de auxílio ou da ajuda de terceiros, acometimento por doenças cardiovasculares limitantes para a prática de exercícios físicos, deficit visual severamente limitante e não compensado por uso de órteses, presença de disfunções neurológicas e ortopédicas de membros inferiores que limitassem a deambulação, alterações otoneurológicas, uso de prótese de membro inferior, participação em outros programas de exercícios físicos e, ao final do processo de intervenção, ter tido frequência inferior a $80 \%$ das sessões.

\section{Tipologia do estudo e sua operacionalização}

0 presente estudo foi desenvolvido por meio de um formato ou desenho quase experimental, considerando-se que esse tipo de pesquisa consiste em determinar um objeto de estudo, selecionar as variáveis que seriam capazes de influenciá-lo e observar os efeitos que a variável independente - aqui representada pelo treinamento sensório-motor - produz diretamente sobre a variável dependente (padrão da marcha de mulheres idosas). Não houve, porém, a designação de um grupo controle para o estudo e não se obedeceu ao critério de aleatoriedade para a escolha dos participantes (14).

Na sua operacionalização foram realizados dois exames da marcha, um antes e outro após a aplicação de 24 sessões de um treinamento, o qual se tipificou como sensório-motor, distribuídas no decorrer de um período de dois meses.

0 tipo de treinamento foi assim descriminado em função de ser realizado por meio da prática diária de exercícios que simulam atividades funcionais, envolvendo força e agilidade, mas propiciando prioritariamente situações que visam ao estado de desequilíbrio, com o intuito de aumentar a resposta neuromotora e promover a estabilidade dinâmico-funcional das articulações $(15,16)$.

Foi adotada uma frequência de três vezes semanais e uma duração de 30 minutos por sessão, repetindo-se as atividades propostas tantas vezes quanto necessário para se completar esse prazo.

0 treinamento foi desenvolvido em um circuito já planejado e instituído pelo PROAFIR, denominado "Circuito de Avaliação e Desenvolvimento da Marcha, do Equilíbrio e do Condicionamento Físico em Idosos", construído em uma sala de 15,84 metros da UNIRG, na qual são dispostas oito estações próprias para a prática de atividades com estímulos proprioceptivos e de equilíbrio, como esquematizadas na Figura 1 e descritas na sequência.

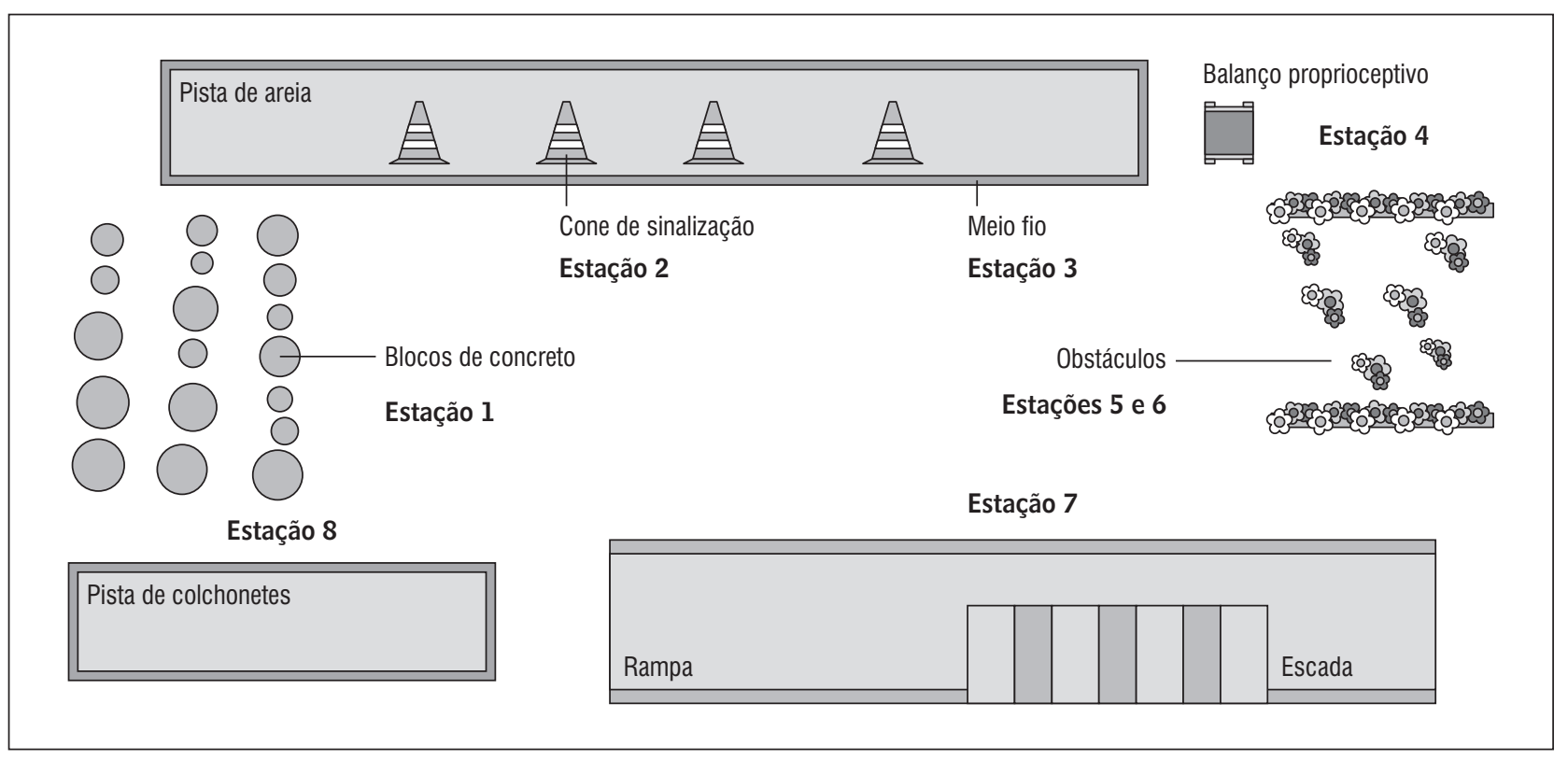

Figura 1 - Esquematização do circuito Fonte: Elaboração dos autores. 
1) Caminhada com ultrapassagem de blocos de concretos em forma de cilindro, com alturas variadas entre 10 a $40 \mathrm{~cm}$ de altura e diâmetro de $35 \mathrm{~cm}$, alternando os membros inferiores, em passada, e apoiando ambos os pés no bloco antes de prosseguir ao próximo.

Os blocos foram organizados em dois níveis de dificuldade, sendo que o primeiro nível tinha sete blocos com pouca variação de altura entre eles. Já o segundo nível foi composto por nove blocos com maiores variações de alturas, garantindo maior intensidade no exercício.

2) Caminhada com ultrapassagem de cinco cones de sinalização de $0,75 \mathrm{~cm}$ de altura, deitados e dispostos a cada 1 metro em uma caixa de areia com extensão de $11 \times 1,10 \mathrm{~m}$.

3) Caminhada sobre meio-fio com 11 metros de comprimento, altura de 10 e 12 centímetros de largura.

4) Apoio bipodal por 20 segundos, sem auxílio das mãos, em balanço proprioceptivo.

5) Caminhada com deslocamentos laterais e frontais entre oito obstáculos presos ao teto por linhas de nylon, organizados em duas colunas de $125 \times 60 \mathrm{~cm}$.

6) Caminhada com deslocamentos e agachamentos sob dois obstáculos com $80 \mathrm{~cm}$ de comprimento, dispostos a uma altura regulada em $130 \mathrm{~cm}$ do solo.

7) Caminhada alternando subida de duas escadas de sete degraus cada uma, com altura de dez centímetros e inclinação de $45^{\circ}$, estruturadas com corrimão a $85 \mathrm{~cm}$ de altura, com descidas em rampas de 3,10 m de extensão.

8) Caminhada em linha reta sobre pista de colchões com comprimento de três metros.

As participantes foram instruídas a realizar o circuito em esforço de intensidade leve, correspondente ao nível 12 da Escala de Borg (17), que demanda, aproximadamente, 55\% da Frequência Cardíaca Máxima, garantindo-lhes a continuidade da atividade pelo tempo estabelecido.

Foram também aconselhadas a utilizarem roupas leves e a realizarem o percurso descalças, com o propósito de evitar possíveis influências induzidas por diferentes tipos de calçados.

No primeiro dia, antes de começar o experimento, as participantes realizaram ambientação prévia por um período suficiente ao reconhecimento e a adaptação ao percurso. Foram informadas que poderiam interromper, a qualquer momento, a execução das atividades diante de algum desconforto percebido por elas. Além disso, foram alertadas a não alterarem suas atividades de vida diária durante o programa de intervenção, pois, assim, reduziriam as possíveis influências de fatores externos sobre os resultados da pesquisa.

No contexto inicial, as participantes foram submetidas a uma análise do padrão da marcha, por meio de técnicas videométricas. Nessa análise, foram considerados os seguintes componentes temporais da marcha: fase de apoio, fase de balanço, duração do ciclo e cadência da marcha (18), e os seguintes componentes espaciais: comprimento da passada e comprimento do passo (19).

Para a realização da filmagem, foi solicitado às participantes que estivessem com uma vestimenta preta e sem calçados. Depois de fornecidas todas as informações sobre o experimento, foi-lhes requerido que caminhassem em passos habituais, com velocidade confortável, em uma pista plana, de superfície regular e reta, de sete metros de comprimento, de modo a ser possível a captura de três ciclos completos da marcha.

O local utilizado para a realização da captura da imagem foi previamente preparado para o ato, tendo sido feitos a eliminação da luz direta sobre a câmera e o enegrecimento do fundo de captura, a fim de evitar que a luz gerada por fontes de iluminação indesejáveis fosse refletida, promovendo os registros de outros objetos que não os de interesse para o exame. Por isso, a parede dentro do plano de captura foi coberta por um tecido preto.

Para a geração das imagens, foi utilizado um modelo de três pontos localizados a $1,3 \mathrm{~cm}$ distal do maléolo tibial, na parte posterior do calcanhar e na cabeça do quinto metatarso (20). Foram utilizados marcadores esféricos reflexivos, com $25 \mathrm{~mm}$, afixados no centro de círculo de PVC preto com $0,5 \mathrm{~mm}$ de espessura e $40 \mathrm{~mm}$ de diâmetro. Tal dispositivo foi utilizado por promover contraste e, assim, comprometer o mínimo possível à precisão da digitalização dos pontos.

A captura das imagens no plano sagital foi realizada por meio de uma câmera filmadora da marca Panasonic, modelo AG-DVC7, posicionada em um tripé a um metro de altura em relação ao solo, e a três metros de distância da pista, de forma que seu eixo ótico ficasse perpendicular ao plano de captura. Para 
a calibragem do sistema, foi utilizado um sistema de quatro pontos não colineares fixados nos vértices de uma das faces de um cubo metálico de $0,50 \mathrm{~m}$ de lado, o qual foi posicionado no centro do setor de registro das imagens, a uma altura equivalente ao valor médio da estatura das participantes. A captura realizada a $30 \mathrm{~Hz}$ possibilitou, no modo entrelaçado, o processamento das imagens a $60 \mathrm{fps}$, o qual foi realizado por meio do software DgeeMe ${ }^{\circledR}$ versão 1.0, no Laboratório de Biomecânica e Comportamento Motor da Universidade Estadual do Rio de Janeiro (UERJ).

No processamento das imagens, foram desprezados o início e o final do trecho filmado, sendo amostrados três ciclos completos da marcha obtidos no registro medial da pista, estratégia adotada para evitar o efeito de aceleração interveniente no comportamento das variáveis cinemáticas em exame.

\section{Ética na pesquisa}

O projeto desta pesquisa foi submetido ao Comitê de Ética em Pesquisa Envolvendo Seres Humanos da Universidade Castelo Branco (UCB/RJ) e aprovado sob o protocolo n. 0002/2009.

A intervenção e coleta de dados aconteceram em conformidade com a Resolução n. 196/96 do Conselho Nacional de Saúde (21). Assim, inicialmente, foi solicitada autorização dos participantes para que o estudo fosse desenvolvido, por meio de um Termo de Consentimento Livre e Esclarecido ao qual se anexou uma carta de esclarecimentos, explicando-se a natureza do estudo.

\section{Tratamento estatístico}

Para se caracterizar o grupo quanto à normalidade de sua distribuição, utilizaram-se os testes de Shapiro-Wilk, e o tratamento estatístico dos dados foi estruturado sob a forma de análise descritiva e inferencial, comum para a análise de dados em pesquisas experimentais e quase-experimentais, concentrando-se na análise descritiva por meio da estimativa de medidas de localização (média e mediana) e dispersão (desvio-padrão e coeficiente de variação) (22).

Foi utilizado o teste de Wilcoxon para a verificação da significância estatística das diferenças dos resultados entre o primeiro e o segundo instantes de mensuração.
Toda a análise inferencial foi desenvolvida tendo, minimamente, 95,00\% de certeza sob a tomada de decisão, ou seja, o nível de significância foi fixado em $5,00 \%(\mathrm{p}<0,05)(23,24)$.

Salienta-se que a característica de grupo de voluntários eliminou a relevância da Potência de Experimento (PPO), enquanto estimativa à discussão do tamanho do grupo de indivíduos investigado. Assim, a PPO somente ratificou a confiabilidade das decisões tomadas (23).

\section{Resultados e discussão}

Na Tabela 1 estão dispostos os valores das variáveis idade, peso, altura e índice de massa corpórea. Após a análise da normalidade de distribuição de acordo com o teste de Shapiro-Wilk, verificou-se que a distribuição foi considerada normal, com valor de $\mathrm{p}>0,05$.

Quanto ao padrão na marcha, na avaliação pré-intervenção, os resultados da Tabela 2 mostram que a média de tempo da fase de apoio contemplou $62 \%$ do ciclo da marcha e a fase de balanço, 38\%, revelando que o grupo, mesmo antes da intervenção,

Tabela 1 - Caracterização do grupo

\begin{tabular}{lcccc}
\hline Idosas & Idade & Peso & Altura & IMC \\
\hline 1 & 75 & 65 & 1,66 & 23,59 \\
2 & 67 & 56 & 1,62 & 21,34 \\
3 & 68 & 46 & 1,55 & 19,15 \\
4 & 68 & 49 & 1,57 & 19,88 \\
5 & 70 & 55 & 1,48 & 25,11 \\
6 & 66 & 65 & 1,70 & 22,49 \\
7 & 66 & 62 & 1,60 & 24,22 \\
8 & 64 & 60 & 1,60 & 23,44 \\
9 & 64 & 55 & 1,63 & 20,70 \\
10 & 75 & 54 & 1,62 & 20,58 \\
11 & 75 & 53 & 1,67 & 19,00 \\
12 & 62 & 56 & 1,59 & 22,15 \\
13 & 63 & 57 & 1,57 & 23,12 \\
14 & 69 & 50 & 1,69 & 17,51 \\
15 & 69 & 63 & 1,68 & 22,32 \\
Valor de p & 0.4835 & 0.6434 & 0.6257 & 0.9278 \\
\hline
\end{tabular}

Fonte: Dados da pesquisa.

Legenda: $p=$ nível de significância; IMC = índice de massa corpórea. 
Tabela 2 - Resultados descritivos das variáveis de cinemática da marcha - 1a mensuração

\begin{tabular}{lcccccc}
\hline Estatística & $\begin{array}{c}\text { Fase de apoio } \\
(\mathbf{s})\end{array}$ & $\begin{array}{c}\text { Fase de balanço } \\
(\mathbf{s})\end{array}$ & $\begin{array}{c}\text { Duração } \\
\text { do ciclo } \\
(\mathbf{s})\end{array}$ & $\begin{array}{c}\text { Comprimento } \\
\text { da passada } \\
(\mathbf{m})\end{array}$ & $\begin{array}{c}\text { Comprimento } \\
\text { do passo } \\
(\mathbf{m})\end{array}$ & $\begin{array}{c}\text { Cadência } \\
\text { (passos/min) }\end{array}$ \\
\hline Média & 70 & 43 & 113 & 1,24 & 0,63 & 106 \\
Desvio-padrão & 07 & 05 & 06 & 0,15 & 0,10 & 06 \\
Mediana & 70 & 45 & 113 & 1,26 & 0,64 & 107 \\
Coeficiente de variação & 06 & 08 & 05 & 8,08 & 9,13 & 05 \\
\hline
\end{tabular}

Fonte: Dados da pesquisa.

Legenda: $\mathrm{s}=$ segundos; $\mathrm{m}=$ metros.

apresentava um padrão de marcha semelhante ao esperado para indivíduos mais jovens, o que justificou a caracterização do grupo como de idosas autônomas $(12,18)$.

Esses achados sugerem uma marcha com qualidade preservada, tendo em vista os resultados encontrados em pesquisa realizada por Winter (11), ao observar que entre 18 idosos a média do tempo de apoio correspondeu a $65,7 \%$ do ciclo e por Teixeira et al. (25), no qual se verificou fases de apoio maiores $(71,42 \pm 5,82 \%$ do ciclo) e de balanço menores (35,97 $\pm 2,90 \%$ do ciclo) em idosos do que em populações mais jovens. Segundo Perry (18), o declínio desse aspecto em idosos é justificado por existir uma necessidade de maior segurança, o que é proporcionado pelo aumento do tempo de duplo apoio, melhorando o equilíbrio. Porém, tal declínio somente pode vir a se instalar em mulheres mais idosas do que as que compuseram o grupo estudado, como sugere Alexander (26), ao relatar que somente $8 \%$ a $19 \%$ dos idosos têm dificuldade na marcha ou necessitam do auxílio de outras pessoas para tal, ou ainda de equipamentos especiais para caminhar, havendo, porém, a tendência de que isso venha a ocorrer com o passar dos anos.

Segundo Santos et al. (27), o exame do perfil da marcha de 25 mulheres sedentárias e praticantes de atividades físicas proporcionou constatar uma cadência com valores médios de 118,36 passos/min. Em relação ao tempo de apoio simples e duplo apoio, obteve-se valor médio de $0,38 \mathrm{~s}$ e $0,26 \mathrm{~s}$, respectivamente; e na variável comprimento do passo, os resultados encontrados foram de $0,54 \mathrm{~m}$.

Em relação ao comprimento do passo, a média encontrada na $1^{\underline{a}}$ mensuração se mostrou um pouco acima dos valores obtidos por Oberg; Karsznia e Oberg (28) que constataram um comprimento do passo de 0,55 $\pm 4,2 \mathrm{~m}$ em mulheres saudáveis, sedentárias com idade entre 60 a 69 anos. Castro et al. (29) estudaram o perfil da marcha de idosas entre 60 e 79 anos, e também encontrou, para o comprimento do passo $(0,46 \pm 8,08 \mathrm{~m})$, valores também inferiores aos obtidos na presente pesquisa.

Tomando-se como parâmetro os achados de estudos de Murray et al. (30), que examinaram 16 idosos, divididos igualmente entre duas faixas etárias - de 60 a 65 e de 63 a 73 anos - os quais apresentaram, respectivamente, valores médios de 1,51 m e 1,36 m de comprimento de passada; e também os de Winter (24), estudo realizado com indivíduos com 68,9 anos de idade, em média, que obteve valores médios de $1,38 \mathrm{~m}$, as idosas investigadas no presente estudo mostraram médias superiores para este indicador.

Já os valores médios encontrados para cadência foram inferiores aos obtidos por Castro et al. (29), que constataram $137,4 \pm 22,64$ passo/min para mulheres de 60 a 79 anos, e aos de Murray, Kory e Clarkson (31), que observaram a média da cadência de 115 passos/minutos ao examinarem 12 idosos com idade entre 60 e 65 anos.

0 grupo apresentou baixa variabilidade (coeficiente de variação $<20,00 \%$ ) nos instantes pré-intervenção (Tabela 2), porém, com aumento deste coeficiente para quase a totalidade das variáveis consideradas, no momento pós-intervenção (Tabela 3), embora continuasse menor que $20 \%$. Isso sugere possíveis efeitos positivos do programa de exercícios estabelecido, para o qual houve respostas diferenciadas, provavelmente por questões genéticas, de saúde e mesmo de outras variáveis intervenientes no resultado.

Como pode ser visto na Tabela 3, após 24 sessões de submissão das idosas ao treinamento, não houve mudanças significativas na cadência, na duração da 
Tabela 3 - Resultados descritivos das variáveis de cinemática da marcha - 2a mensuração

\begin{tabular}{lcccccc}
\hline Estatística & $\begin{array}{c}\text { Fase de apoio } \\
(\mathbf{s})\end{array}$ & $\begin{array}{c}\text { Fase de balanço } \\
(\mathbf{s})\end{array}$ & $\begin{array}{c}\text { Duração } \\
\text { do ciclo } \\
(\mathbf{s})\end{array}$ & $\begin{array}{c}\text { Comprimento } \\
\text { da passada } \\
(\mathrm{m})\end{array}$ & $\begin{array}{c}\text { Comprimento } \\
\text { do passo } \\
(\mathrm{m})\end{array}$ & $\begin{array}{c}\text { Cadência } \\
\text { (passos/min) }\end{array}$ \\
\hline Média & 70 & 42 & 112 & 1,32 & 0,66 & 107 \\
Desvio-padrão & 04 & 04 & 06 & 0,10 & 0,06 & 06 \\
Mediana & 68 & 43 & 113 & 1,31 & 0,68 & 106 \\
Coeficiente de variação & 09 & 11 & 05 & 11,27 & 15,79 & 05 \\
\hline
\end{tabular}

Fonte: Dados da pesquisa.

Legenda: $\mathrm{s}=$ segundos; $\mathrm{m}=$ metros.

fase de apoio e de balanço, e mesmo no comprimento do passo.

Porém, a aplicação do teste de Wilcoxon (Tabela 4) demonstrou ser estatisticamente significativa a diferença entre pré e pós-teste, no que se refere ao comprimento da passada. Infere-se que isso possa ter ocorrido em função da necessidade de transposição dos obstáculos dentro do circuito, o que condiz com pesquisa realizada com 45 idosas sedentárias e ativas com média de idade de 73,6 anos $( \pm 9,18)$, que foram submetidas a um treinamento em um percurso de 8 m com transposição de obstáculos, no qual os autores verificaram que o comprimento e a velocidade da passada que precede a ultrapassagem do obstáculo foram alterados para aumentar a segurança (32).

Tabela 4 - Resultados dos Testes de Wilcoxon (Pré × Pós)

\begin{tabular}{llc}
\hline Lado & Variável & Valor-p \\
\hline Direito & Fase de apoio & 0,85 \\
& Fase de balanço & 0,32 \\
& Duração do ciclo & 0,71 \\
& Comprimento da passada & $0,02^{*}$ \\
& Comprimento do passo & 0,23 \\
& Cadência & 0,91 \\
\hline
\end{tabular}

Fonte: Dados da pesquisa.

\section{Conclusão}

Concluiu-se que o treinamento sensório-motor, muito embora possa melhorar o equilíbrio do indivíduo idoso quando a instabilidade é observada em posição ortostática, trazendo-lhe segurança para desenvolver a marcha e, consequentemente, as atividades da vida diária, pode não afetar diretamente tal função nos seus aspectos biomecânicos. No entanto, para conclusões mais categóricas, propõe-se que novos estudos sejam realizados com a avaliação de outros aspectos do padrão da marcha dos sujeitos, tais como deslocamento látero-lateral de tronco, rotação da cintura pélvica, rotação da cintura escapular, deslocamento de membros superiores, movimento de cabeça e equilíbrio nas mudanças de direção.

\section{Referências}

1. Vandervoort AA. Alterações biológicas e fisiológicas. In: Pickles B, Compton A, Cott C, Simpson J, Vandervoort A. Fisioterapia na terceira idade. 2a ed. São Paulo: Santos Livraria; 2000.

2. Konrad HR, Girardi M, Helfert R. Balance and aging. Laryngoscope. 1999;109(9):1454-60.

3. Pereira LSM, Basques FV, Marra TA. Avaliação da marcha em idosos. Mundo Saúde. 1999;23(4):221-9.

4. Matsudo SM, Matsudo VKR, Barros TL Neto. Impacto do envelhecimento nas variáveis antropométricas, neuromotoras e metabólicas da aptidão física. Rev Bras Ciên Mov. 2000;8(4):21-32.

5. Lustri WR, Morelli JGS. Aspectos biológicos do envelhecimento. In: Rebelatto JR, Morelli JGS. Fisioterapia geriátrica: a prática da assistência ao idoso. São Paulo: Manole; 2004.

6. Souza RR. Anatomia do envelhecimento. In: Papaléo M Netto, de Carvalho ET Filho. Geriatria: fundamentos, clínica e terapêutica. São Paulo: Atheneu; 2006. 
7. Papaléo M Netto, Britto MFC. Urgências em geriatria. São Paulo: Atheneu; 2001.

8. Verdú E, Ceballos D, Vilches JJ, Navarro X. Influence of aging on peripheral nerve function and regeneration. J Peripher Nerv Syst. 2000;5(4):191-208.

9. Xu E, Hong Y, Li J, Chan K. Effect of tai chi exercise on proprioception of ankle and knee joints in old people. Br J Sports Med. 2004;38(1):50-4.

10. Imms FJ, Edholm OG. Studies of gait and mobility in the elderly. Age \& Ageing. 1981;10:147-156.

11. Wall JC. A marcha. In: Durward BR, Baer GD, Rowe PJ. Movimento funcional humano: mensuração e análise. São Paulo: Manole; 2001.

12. Paixão CM Jr, Heckmann M. Distúrbio da postura, marcha e quedas. In: Freitas EV, Py L, Néri AL, Cançado FAX, Gorzoni FAX, Rocha S. Tratado de geriatria e gerontologia. Rio de Janeiro: Guanabara Koogan; 2002.

13. Ruwer SL, Rossi AG, Simon LF. Equilíbrio no idoso. Rev Bras Otorrinolaringol. 2005;71(3):298-303.

14. Campbell DT, Stanley JC. Delineamentos experimentais e quase-experimentais de pesquisa. São Paulo: EPU/EDUSP; 1979.

15. Lephart SM, Pincivero DM, Giraldo JL, Fu FH. The role of proprioception in the management and rehabilitation of athletic injuries. Am J Sports Med. 1997; 25(1):130-7.

16. Souza ACS, Paiva PB, Guth VJ, Martins AC, Santos GM, Mazo, GJ. 0 efeito do treinamento sensório-motor no equilíbrio de idosas. Revista Digital - Buenos Aires. 2009 [acesso 10 jun. 2010]. Disponível em: http:// www.efdeportes.com.

17. Borg GAV, Noble BJ. Perceived exertion. In: Wilmore JH. Exercise and sport sciences reviews. New York: Academic Press; 1974. p. 131-57.

18. Perry J. Análise de marcha. São Paulo: Manole; 2005.

19. Vaughan CL, Davis BL, O' Connor JC. Dynamics of human gait. 2nd ed. Cape Town, South Africa: Kiboho Publisher; 1999.

20. Batista LA, Faro AM, Torres S, Itaborahy AS. Comportamento temporal do movimento. Revista Inuaf Studia Scientiae Rerum Diffusio, Lolé. 2003;3(5):70-85.
21. Brasil. Ministério da Saúde. MS. Conselho Nacional de Saúde MS. Resolução196/96. Diretrizes e Normas Regulamentadoras de Pesquisa Envolvendo Seres Humanos. Diário Oficial da República Federativa do Brasil, Poder Legislativo, 10 out. 1996.

22. Costa PLO Neto. Estatística. São Paulo: Edgard Blücher; 2002.

23. Bunchaft G, Kellner SRO. Estatística sem mistérios. São Paulo: Edgard Blücher; 1999.

24. Winter DA. The biomechanics and motor control of human gait: normal, elderly and pathological. 2a ed. Waterloo: University of Waterloo Press; 1991.

25. Teixeira CS, Link DM, Ribeiro JK, Costa VP, Mota CB. Aspectos biomecânicos do caminhar em idosos. Anais Jornada Acadêmica Integrada, Santa Maria. Santa Maria: UFSM; 2002.

26. Alexander NB. Gait disorders in older adults. J Am Geriatr Soc. 1996;44(4):434-51.

27. Santos DM, Melo SIL, Carneiro CC, Andrade MC. Características da marcha de idosos considerando a atividade física e o sexo. Fisioter Mov. 2008;21(4):137-48.

28. Oberg T, Karsznia A, Oberg K. Basic gait parameters: reference data for normal subjects, 10-79 years of age. J Rehabil Res Dev. 1993;30(2):210-31.

29. Castro CLN, Santos JACB, Leifeld PS, Bizzo LV, Silva LC, Almeida TF, et al. Estudo da marcha em idosos: resultados preliminares. Acta Fisiátr. 2000;7(3):103-7.

30. Murray MP, Kory RC, Clarkson BH. Walking patterns in healthy old men. J Gerontol. 1969;24(2):169-78.

31. Murray MP, Drought BJ, Kory RC. Walking patterns of normal men. J Bone Joint Surg.1964;46:335-60.

32. Cozzani M, Castro EM. Estratégias adaptativas durante o andar de idosos institucionalizados. In: Anais X Congresso Brasileiro de Biomecânica Ouro Preto: UFMG; 2003.

Recebido: 02/07/2011

Received: 07/02/2011

Aprovado: 25/10/2011

Approved: 10/25/2011 